\title{
BMJ Open Acupuncture for erectile dysfunction: a systematic review protocol
}

\author{
Xiaoming Cui, ${ }^{1,2}$ Xiaoli Li, ${ }^{2}$ Weina Peng, ${ }^{1}$ Jing Zhou, ${ }^{1,2}$ Jinna $Y u,{ }^{1}$ \\ Yongming Ye, ${ }^{1}$ Zhishun Liu ${ }^{1}$
}

To cite: Cui X, Li X, Peng W, et al. Acupuncture for erectile dysfunction: a systematic review protocol. BMJ Open 2015;5:e007040

doi:10.1136/bmjopen-2014007040

- Prepublication history for this paper is available online. To view these files please visit the journal online (http://dx.doi.org/10.1136/ bmjopen-2014-007040).

Received 28 October 2014 Revised 16 February 2015 Accepted 17 February 2015

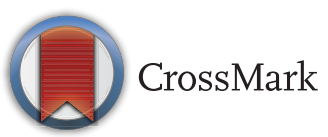

${ }^{1}$ Department of Acupuncture, Guang'anmen Hospital, China Academy of Chinese Sciences, Beijing, China ${ }^{2}$ Beijing University of Chinese Medicine, Beijing, China

Correspondence to Professor Zhishun Liu; liuzhishun@aliyun.com

\section{ABSTRACT}

Introduction: This systematic review protocol aims to provide a protocol for assessing the safety and effectiveness of acupuncture for the treatment of erectile dysfunction(ED). Previous systematic reviews did not draw convincing conclusions owing to high heterogeneity and few included randomised controlled trials, so it is necessary to reassess the efficacy and safety of acupuncture for ED.

Methods and analysis: Eight electronic databases will be searched: the Cochrane Central Register of Controlled Trials (CENTRAL), MEDLINE, PubMed, EMBASE, PsycInfo, the Chinese Biomedical Literature Database (CBM), the Chinese Medical Current Content (CMCC) and the China National Knowledge Infrastructure (CNKI). Related Chinese literature will be searched in other Chinese databases. All relevant randomised controlled trials in English or Chinese without any restrictions of publication type will be included. The main outcome measure will be improvements in sexual activity assessed by validated questionnaires. Assessment of risk of bias, data synthesis and subgroup analysis will be carried out using Review Manager 5.3.

Ethics and dissemination: The results of the systematic review will be disseminated via publication in a peer-reviewed journal and presented at a relevant conference. The data we will use do not include individual patient data, so ethical approval is not required.

Trial registration number: PROSPERO CRD42014013575.

\section{INTRODUCTION}

\section{Description of the condition}

Erectile dysfunction (ED) is defined as the persistent inability to achieve or maintain an erection sufficient to permit satisfactory sexual performance. ${ }^{12}$ Epidemiological study found that the prevalence of ED is high; for example, it affects approximately $22 \%$ of US men. ${ }^{3}$ About 150 million individuals are estimated to suffer from ED worldwide. ${ }^{45}$ As the life expectancy of the general population increases, the healthcare burden and quality-of-life issues associated with $\mathrm{ED}$ are expected to be considerable. ${ }^{6}$

\section{Strengths and limitations of this study}

- Study selection, data extraction and quality assessment will be performed independently by two researchers, which will ensure that all relevant studies are included without personal biases.

- Medical databases in other languages (eg, Korean and Japanese) will not be searched because of language barriers, so language bias may exist.

- There may be high heterogeneity from the various evaluation standards in different acupuncture therapies.

The pathophysiology of ED may include arterial, neurogenic, hormonal, cavernous, iatrogenic and psychogenic causes. ${ }^{7}$ ED can also result from a generalised vascular disorder caused by endothelial dysfunction. ${ }^{8}$ A comprehensive evaluation for ED consists of a complete medical and sexual history, a physical examination, a psychological assessment and appropriate laboratory tests. ${ }^{9}$

The current therapies for ED include oral pharmacotherapy, intracavernous injections and implantation of a penile prosthesis. ${ }^{10}$ Phosphodiesterase type 5 inhibitors such as sildenafil are currently used as the first-line treatments for ED. ${ }^{11}$ However, there is still a significant population of patients who remain refractory to this therapy. ${ }^{12}$ For patients treated with intracavernous injections, $29-41 \%$ have mild penile pain. ${ }^{10} 13$ Moreover, implantation of a penile prosthesis is expensive, and may cause penile deformity. ${ }^{10}$ Therefore, an ideal treatment for ED has not as yet been developed.

\section{Description of the intervention}

Traditional medicine (TM) is increasingly accepted by people in the developing and developed world as an alternative to conventional treatments. ${ }^{14}$ One-third of American residents seek service from TM practitioners every year for illnesses that do not respond to conventional treatment. ${ }^{15}$ Acupuncture, which involves the insertion of fine needles into the skin at specific points, has a long 
history of use in China and is one of the important treatments used in TM.

\section{How the intervention might work}

Traditional Chinese medicine (TCM), which is an important part of TM, is based on the theoretical concepts of Yin-Yang and the five elements, and theorises that health is maintained by a balance of energy within the body. Acupuncture helps to correct imbalances to relieve symptoms by stimulating various meridian points. The equilibrium of the autonomic nervous system is the modern equivalent to Yin-Yang balance. ${ }^{16}$ Acupuncture may positively affect the pathophysiology of ED based on its homeostatic influence on the autonomic nervous system. ${ }^{17}$ Many in vivo studies demonstrate that neuropeptides, which are vital to the central control of male sexual behaviour, are involved in the mechanisms of pain relief after acupuncture. ${ }^{18-20}$ Moreover, acupuncture might modulate nitric oxide, which is related to the treatment of ED. ${ }^{2}$ Some studies indicate a high success rate in patients with ED after TCM treatment, including acupuncture. ${ }^{21-24}$

\section{OBJECTIVES}

This article describes the protocol for a systematic review that will assess the evidence for the effectiveness and safety of acupuncture for ED.

\section{METHODS AND ANALYSIS}

\section{Criteria for considering studies for this review}

Types of studies

All relevant randomised controlled trials (RCTs) in English and Chinese without any restrictions on publication type will be included and quasi-RCTs will be excluded.

\section{Types of participants}

Studies evaluating men aged more than 18 years of any ethnic background and nationality will be included. ED must be diagnosed by clinical and/or instrumental methods. The diagnosis will be based on the Diagnostic and Statistical Manual of Mental Disorders Third Edition (DSM)-III, DSM-III-R, DSM-IV, International Statistical Classification of Diseases and Related Health Problems (ICD)-10 criteria or any other described criteria. Patients with drug-induced ED, external genital malformation or organic damage to the genitourinary system such as pudendal nerve injury from trauma or surgery will be excluded.

\section{Types of interventions}

Acupuncture type can include: body acupuncture, electroacupuncture, scalp acupuncture, ear acupuncture, fire needling, elongated needle, intradermal needling or dry needling. Laser acupuncture and point injection will be excluded. The control intervention can include: no treatment, placebo/sham acupuncture or other interventions (eg, drugs, physical therapy). Trials that evaluate acupuncture plus another therapy compared with the other therapy alone will also be included. Trials that only compare different types of acupuncture or different points will be excluded.

\section{Types of outcome measures}

\section{Primary outcomes}

The primary outcome will be improvement in sexual activity. This will be assessed through validated questionnaires such as the International Index of Erectile Function (IIEF). ${ }^{25}$ Trials with non-validated questionnaires or no clear descriptions of evaluation methods will be excluded.

\section{Secondary outcomes}

1. Quality of life

2. Satisfaction with the treatment

3. Improvement in depression or anxiety indices

4. Safety assessment as measured by incidence and severity of adverse effects (eg, pain or dizziness)

\section{Search methods for identification of studies} Electronic searches

An electronic search strategy will be designed to search relevant references in the Cochrane Central Register of Controlled Trials (CENTRAL), MEDLINE, PubMed, EMBASE, PsycInfo, the Chinese Biomedical Literature Database (CBM), the Chinese Medical Current Content (CMCC) and the China National Knowledge Infrastructure (CNKI). The search will be performed in English and Chinese. The following terms will be used: acupuncture, acupuncture therapy, electroacupuncture, fire needling, elongated needle, intradermal needling, dry needling, erectile dysfunction, impotence, erection failure, male sexual dysfunction, randomised controlled trials, double-blind method and single-blind method (table 1 details of the search strategy for EMBASE). The search terms will be translated into Chinese when reviewers search the Chinese databases. The following literature sources in Chinese will also be searched: dissertations in CNKI, and conference papers in the China Conference Paper Database. Relevant references cited in selected studies will also be searched.

\section{Searching other resources}

Reference texts including andrology textbooks, integrative/alternative and complementary medicine textbooks and clinical guidelines for relevant trials will also be searched manually.

\section{Data collection and analysis}

Selection of studies

The abstracts of all publications will be independently screened by the review authors (XC and JZ). The full text of articles potentially suitable for the review will be obtained to assess relevance based on the inclusion/ 


\begin{tabular}{ll} 
Table 1 & EMBASE search strategy \\
\hline No & Searching item \\
\hline 1 & Clinical article \\
2 & Clinical study \\
3 & Clinical trial \\
4 & Controlled study \\
5 & Randomized controlled trials \\
6 & Major clinical study \\
7 & Double blind method \\
8 & Multicenter study \\
9 & Single blind method \\
10 & Crossover procedure \\
11 & Placebo \\
12 & Or/1-11 \\
13 & Erectile dysfunction \\
14 & Impotence \\
15 & Erection failure \\
16 & Male sexual dysfunction \\
17 & Penis erection \\
18 & Or/13-17 \\
19 & Exp ACUPUNCTURE \\
20 & acupuncture.tw \\
21 & electroacupuncture.tw. \\
22 & Exp Acupuncture Therapy \\
23 & Fire needling \\
24 & Elongated needle \\
25 & Intradermal needling \\
26 & Scalp acupuncture \\
27 & Ear acupuncture \\
28 & Dry needling \\
29 & Or/19-28 \\
30 & 12 and 18 and 29 \\
\hline & \\
&
\end{tabular}

exclusion criteria. We will discuss with ZL if there are any discrepancies. The studies that do not fulfil the inclusion criteria will be excluded and listed with reasons for their exclusion.

\section{Data extraction and management}

Data for the trials will be extracted independently by two review authors (XC and JZ) using a standard form. The following information will be included.

1. Study methods and characteristics (design, method of randomisation, inclusion/exclusion criteria and withdrawals/dropouts).

2. Participants (number of participants, age range, diagnostic criteria, disease course).

3. Intervention (type of acupuncture therapy, duration of session).

4. Control (no treatment, placebo therapy or other active treatment).

5. Outcomes (types of outcome measures, reported outcomes, adverse events, follow-up time and results).

Extracted data will be compared by two review authors for completeness and accuracy and double-checked by another review author if necessary. Disagreements will be solved through discussion with ZL. New data will be transferred into Review Manager 5.3. ${ }^{26}$

\section{Assessment of risk of bias in included studies}

The risk of bias in the included studies will be assessed independently by two authors (XC and JZ) and presented in a risk of bias table. Decisions will be made based on the domains and criteria of the Cochrane Collaboration's tooll ${ }^{27}$ for assessing risk of bias. The following domains will be assessed:

1. Selection bias: random sequence generation and allocation concealment.

2. Performance bias: blinding of investigators, participants and care providers.

3. Detection bias: blinding of outcome assessment.

4. Attrition bias: incomplete data/differential dropout.

5. Reporting bias: selective reporting.

6. Other bias: for example, conflicts of interest, follow-up, non-intention-to-treat or per-protocol analysis.

For each domain, the following description will be used to assess proper management of the risk of bias: 'low risk,' 'high risk,' or 'unclear.' We will grade the quality of included studies and risk of bias using the Grading of Recommendations Assessment, Development and Evaluation (GRADE) tool $^{28}$ with GRADEprofiler (GRADEpro) V.3.6 software. Any disagreements will be resolved by discussion with ZL.

\section{Measures of treatment effect}

For continuous data, the mean difference (MD) will be used to measure treatment effect with $95 \%$ CIs. In case outcome variables have different scales, the standardised mean difference will be used with $95 \%$ CIs.

For dichotomous data, treatment effects are presented as a risk ratio (RR) with $95 \%$ CIs. Other binary data will be changed into the RR form.

\section{Unit of analysis issues}

The unit of analysis will be each patient recruited into the trials.

\section{Dealing with missing data}

For each included study, the number of dropouts, exclusions from the analysis and missing data will be gathered by contacting the study author. If we fail to obtain sufficient data, we will assume dichotomous outcomes for patients not experiencing any change in their clinical outcome variables. We will then perform sensitivity analyses to assess how sensitive the results are to changes in the assumptions made.

If necessary, the potential impact of missing data on the findings of the review will be described in the 'Discussion' section.

\section{Assessment of heterogeneity}

Cochran's $Q$ test ${ }^{29}$ will be performed for the detection of heterogeneity. The $\mathrm{I}^{2}$ statistic will be used to measure heterogeneity among the trials in each analysis. An $\mathrm{I}^{2}$ value of $50 \%$ or more indicates a substantial level of inconsistency. If we identify substantial heterogeneity, we 
will report it and explore possible causes using subgroup analyses.

\section{Assessment of reporting biases}

If we are able to pool data from more than 10 trials for the primary outcome, a funnel plot will be created and examined to explore possible small study biases. We will interpret results carefully based on several explanations for funnel plot asymmetry.

\section{Data synthesis}

If two or more eligible RCTs are identified, metaanalyses will be performed with Review Manager 5.3. Whether a fixed effects model or a random effects model will be used depends on the results of the $\chi^{2}$ test and $\mathrm{I}^{2}$ test for heterogeneity. If substantial statistical heterogeneity is found, we will adopt a random effects model. If no substantial statistical heterogeneity is detected $\left(\mathrm{I}^{2}<50 \%\right)$, we will use a fixed effects model. If clinical and methodological heterogeneity is present, we will perform subgroup analyses. If not, we will not pool the data but conduct a systematic narrative synthesis providing information to summarise and explain the characteristics and findings of the included studies.

Subgroup analysis and investigation of heterogeneity

We plan to carry out the following subgroup analyses, if possible.

1. Comparison between acupuncture and sham, placebo or no treatment.

2. Comparison between acupuncture and routine Western medicine treatment.

3. Comparison between manual acupuncture and electroacupuncture.

4. Comparison between psychosocial ED and physiological ED.

We will use the formal test for subgroup interactions in Review Manager 5.3.

\section{Sensitivity analysis}

Sensitivity analysis will be conducted to explore the effects of trial risk of bias on important outcomes. In the

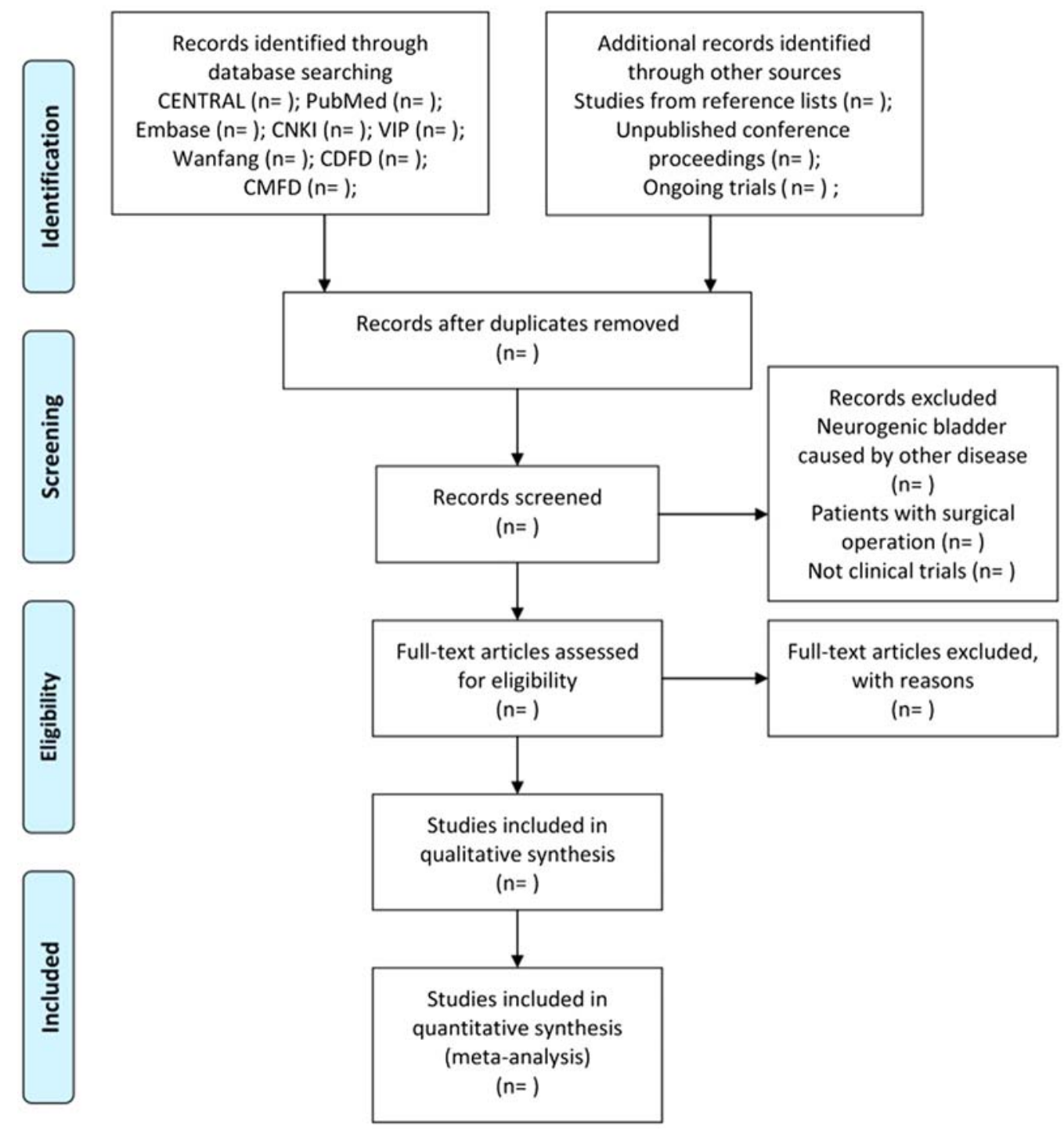

Figure 1 Process of the systematic review. 
analysis, we will exclude lower quality trials and compare the results with those using the worst-case strategy to combine studies. Then we will have a discussion to decide whether the lower quality trials should be excluded, depending on their sample size, strength of evidence and influence on pooled effect size.

\section{DISCUSSION}

The previous review which was published 6 years ago failed to determine if there are beneficial effects of acupuncture therapy in ED treatment. ${ }^{30}$ Nearly $30-40$ RCTs have been published within the past 6 years on acupuncture and ED, so it is necessary to reassess the efficacy and safety of acupuncture for ED.

The flow chart of this systematic review is shown in figure 1. This review will be helpful to clinicians treating ED and may provide evidence for researchers. Patients with ED may also benefit from potential alternative interventions.

However, this systematic review will have some limitations. The medical databases in other languages (eg, Korean and Japanese) will not be covered because of language barriers, so a language bias may exist. High heterogeneity may also arise from the various evaluation standards from different acupuncture therapies. Nevertheless, this systematic view should help further expand our understanding of acupuncture treatments for ED

Acknowledgements The authors would like to thank Tyler Calway for helping them to revise the English language of the manuscript.

Contributors $X C$ and $Z L$ contributed to the conception of the study. The manuscript protocol was drafted by $X C$, and was revised by $X L, W P, J Y$, YY and ZL. The search strategy was developed by all of the authors, and will be performed by XC and JZ, who will also independently screen the potential studies, extract data of included studies, assess the risk of bias and complete the data synthesis. ZL will arbitrate the disagreements and ensure that no errors are introduced during the study. All authors approved the publication of the protocol.

Competing interests None.

Patient consent Obtained.

Provenance and peer review Not commissioned; externally peer reviewed.

Data sharing statement The technical appendix, statistical code and data set are available from the corresponding author at Dryad repository, who will provide a permanent, citable and open access home for the data set.

Open Access This is an Open Access article distributed in accordance with the Creative Commons Attribution Non Commercial (CC BY-NC 4.0) license, which permits others to distribute, remix, adapt, build upon this work noncommercially, and license their derivative works on different terms, provided the original work is properly cited and the use is non-commercial. See: http:// creativecommons.org/licenses/by-nc/4.0/

\section{REFERENCES}

1. NIH Consensus. NIH Consensus Conference. Impotence. NIH Consensus Development Panel on Impotence. JAMA 1993;270:83-90.

2. Lue TF. Erectile dysfunction. N Engl J Med 2000;24:1802-13.
3. Rosen RC, Fisher WA, Eardley I, et al. The multinational Men's Attitudes to Life Events and Sexuality (MALES) study: I. Prevalence of erectile dysfunction and related health concerns in the general population. Curr Med Res Opin 2004;20:607-17.

4. Aytaç, Mckinlay, Krane. The likely worldwide increase in erectile dysfunction between 1995 and 2025 and some possible policy consequences. BJU Int 1999;84:50-6.

5. McKinlay JB. The worldwide prevalence and epidemiology of erectile dysfunction. Int J Impot Res 2000;12:S6-11.

6. Tsertsvadze A, Yazdi F, Fink HA, et al. Diagnosis and treatment of erectile dysfunction. AHRQ Evidence Report/Technology Assessment no. 171. Bethesda, MD: Agency for Healthcare Research and Quality; May 2009. AHRQ publication no. 08 (09)-E016. Accessed.

7. De Boer BJ, Bots ML, Lycklama a Nijeholt AA, et al. Erectile dysfunction in primary care: prevalence and patient characteristics. The ENIGMA study. Int J Impot Res 2004;16:358-64.

8. Sullivan ME, Keoghane SR, Miller MA. Vascular risk factors and erectile dysfunction. BJU Int 2001;87:838-45.

9. Carbone DJ Jr, Seftel AD. Erectile dysfunction. Diagnosis and treatment in older men. Geriatrics 2002;57:18-24.

10. Hatzimouratidis K, Amar E, Eardley I, et al. Guidelines on male sexual dysfunction: erectile dysfunction and premature ejaculation. Eur Urol 2010;57:804-14.

11. Hatzimouratidis $\mathrm{K}$, Hatzichristou DG. A comparative review of the options for treatment of erectile dysfunction. Drugs 2005;65:1621-50.

12. Lasker GF, Maley JH, Kadowitz PJ. A review of the pathophysiology and novel treatments for erectile dysfunction. Adv Pharmacol Sci 2010;2010:pii:730861.

13. Padma-Nathan H, Hellstrom WJG, Kaiser F E, et al. Treatment of men with erectile dysfunction with transurethral alprostadil. $N$ Engl $J$ Med 1997;336:1-7.

14. Traditional Medicine Growing Needs and Potential-WHO Policy Perspectives on Medicines, No. 002, May 2002.

15. Claxton M. Culture, health and civilization. In: Leander B, ed. Culture and Health. Paris: UNESCO Pub, 1996:17-46.

16. Kho HG, Sweep CGJ, Chen X, et al. The use of acupuncture in the treatment of erectile dysfunction. J Urol 1999;162:630-1.

17. Tabeeva DM. Autonomic nervous system function of alcoholics during acupuncture treatment. Zhurnal Nevropatol Psikhiatr Im SS Korsakova 1988;88:33-6.

18. Carmichael MS, Humbert R, Dixen J, et al. Plasma oxytocin increases in the human sexual response. J Clin Endocrinol Metab 1987;64:27-31.

19. Dornan WA, Malsbury CW. Neuropeptides and male sexual behavior. Neurosci Biobehav Rev 1989;13:1-15.

20. Kho HG, Robertson EN. The mechanisms of acupuncture analgesia: review and update. Am J Acupunct 1997;25:261-81.

21. Yaman LS, Kiliç $S$, Sarica K, et al. The place of acupuncture in the management of psychogenic impotence. Eur Urol 1993;26:52-5.

22. Wu JZ, Zhang Q, Wu WC, et al. 100 Cases of impotence treated by acupuncture and moxibustion. J Trad Chin Med 1989;9:184-5.

23. Li Z, Ye C. Treating impotence with traditional Chinese medicine coordinated by acupuncture and moxibustion. J Trad Chin Med 1988;8:121-2.

24. Ahn SY. Saam-Ohang acupuncture treatment methods of impotence in male sex clinic. J Orient Med 1996;1:200-2.

25. Rosen RC, Cappelleri JC, Gendrano N III. The International Index of Erectile Function (IIEF): a state-of-the-science review. Int J Impot Res 2002;14:226-44.

26. Not Available, Review Manager, 5.3 for Windows. Oxford, England Cochrane Collaboration, 1999.

27. Higgins JPT, Green S. Cochrane handbook for systematic review of intervention version 5.1.0 [updated March 2011]. The Cochrane Collaboration, 2011. http://www.cochrane-handbook.org (accessed Dec 2014).

28. Guyatt GH, Oxman AD, Vist GE, et al. GRADE: an emerging consensus on rating quality of evidence and strength of recommendations. BMJ 2008;336:924-6.

29. Pbert L, Adams A, Quirk M, et al. The patient exit interview as an assessment of physician-delivered smoking intervention: a validation study. Health Psychol 1999;18:183-8.

30. Lee MS, Shin BC, Ernst E. Acupuncture for treating erectile dysfunction: a systematic review. BJU Int 2009;104:366-70. 